\title{
Comparativo de casos de violência sexual contra criança e adolescente no período
}

\section{8-2020}

\author{
Comparison of cases of sexual violence against children and adolescents in the period 2018-2020
}

Comparación de casos de violencia sexual contra niños, niñas y adolescentes en el período 2018-

Renata Kelly Nogueira Trajano ORCID: https://orcid.org/0000-0002-3571-1713 Universidade de Pernambuco, Brasil

E-mail: renatatrajano.odonto@gmail.com

Carolina Viana Vasco Lyra

ORCID: https://orcid.org/0000-0002-5474-6815

Universidade de Pernambuco, Brasil

E-mail: carol-lyra98@hotmail.com

Tamyres Yasmin Goes e Sá

ORCID: https://orcid.org/0000-0002-2647-2837

Universidade de Pernambuco, Brasil E-mail: tamyres.yasmin@upe.br

Ana Cláudia Amorim Gomes

ORCID: https://orcid.org/0000-0003-0934-6086

Universidade de Pernambuco, Brasil

E-mail: anacagomes@upe.br

\begin{abstract}
Resumo
Esse estudo objetiva comparar casos de violência sexual contra crianças e adolescentes no período de 2018-2020. Tratase de estudo documental, analítico, tendo como fonte de informações laudos periciais de violência sexual produzidos no Instituto Médico Legal de uma capital do Nordeste de vítimas com idade entre 0 e 18 anos. O estudo compreende o período entre os meses de abril a junho dos anos de 2018 a 2020. Identificou-se 309 laudos de violência sexual no período do estudo. Houve, no ano de 2020, um aumento no percentual de vítimas do sexo masculino, na faixa etária de 0 a 11 anos, que foram violentadas em seus domicílios, por familiares. Observou-se, em 2020, declínio de 44,8\% nos laudos de violência sexual quando comparado ao mesmo período de 2019, e 37,3\% quando comparado a 2018. O estudo aponta alta prevalência de violência sexual infantojuvenil, com aumento em 2020 do percentual de agressores com vínculo intrafamiliar com a vítima e do domicílio como local de ocorrência da violência. O quantitativo de laudos periciais diminuiu expressivamente no ano de 2020, possivelmente devido ao fechamento de escolas e restrições dos serviços públicos de saúde e assistência voltados para essa população devido a pandemia da Covid-19.
\end{abstract}

Palavras-chave: Delitos sexuais; Criança; Adolescente; Infecções por coronavirus.

\begin{abstract}
This study aims to compare cases of sexual violence against children and adolescents during the period of 2018-2020. This is a documentary research, analytical study, having as source of information expert reports of sexual violence produced at the Medico-Legal Institute of a capital from the Northeast in Brazil. Addressing victims aged between 0 and 18 years. The study covers from April to June of 2018 until 2020. 309 reports of sexual violence were identified during this study. In 2020, there was an increase in the percentage of male victims, aged 0 to 11 years, who were raped in their homes by family members. In 2020 , there was a decline of $44.8 \%$ in reports of sexual violence when compared to the same period frame in 2019 , and $37.3 \%$ when compared to 2018 . The study points out to a high prevalence of child and youth sexual violence, with an increase in 2020 the percentage of aggressors with an intrafamily bond with the victim and the home as the place where the violence occurred. The number of expert reports decreased significantly in 2020, possibly due to the closure of schools and restrictions on public health care services aimed at this population due to the Covid-19 pandemic.
\end{abstract}

Keywords: Sexual offenses; Child; Adolescent; Coronavirus infections.

\section{Resumen}

Este estudio tiene como objetivo comparar los casos de violencia sexual contra niños, niñas y adolescentes en el período 2018-2020. Se trata de un estudio documental, analítico, que tiene como fuente de información los informes periciales sobre violencia sexual elaborados en el Instituto Médico Legal de una capital del Nordeste de víctimas de entre 0 y 18 años. El estudio cubre el período de abril a junio de 2018 a 2020. Se identificaron 309 denuncias de violencia sexual durante el período de estudio. En 2020, hubo un aumento en el porcentaje de víctimas masculinas, de 0 a 11 años, que 
fueron violadas en sus hogares por familiares. En 2020, se observó una disminución del 44,8\% en las denuncias de violencia sexual en comparación con el mismo período en 2019, y del 37,3\% en comparación con 2018. El estudio apunta a una alta prevalencia de violencia sexual infantil y juvenil, con un aumento en 2020 el porcentaje de agresores con vínculo intrafamiliar con la víctima y el hogar como lugar donde ocurrió la violencia. La cantidad de informes periciales disminuyó significativamente en 2020, posiblemente debido al cierre de escuelas y restricciones en los servicios de salud pública y asistencia dirigidos a esta población debido a la pandemia Covid-19.

Palabras clave: Delitos Sexuales; Niño; Adolescente; Infecciones por coronavirus.

\section{Introdução}

A violência sexual é qualquer ato sexual, tentativa de obter um ato sexual, comentários ou insinuações sexuais não desejados, atos de tráfico ou dirigidos contra a sexualidade de uma pessoa usando coerção, por qualquer pessoa, independente de sua relação com a vítima, em qualquer contexto, incluindo casa e trabalho, mas não limitado a estes (OMS, 2015). Esse tipo de violência ocorre de forma insidiosa e persistente, acometendo crianças de todas as idades, etnias e culturas. Estima-se que, mundialmente, 225 milhões de crianças e adolescentes são vítimas de abusos sexuais anualmente (Pinheiro, 2006).

Estudos alertam que a violência sexual ocorre majoritariamente contra pessoas do sexo feminino, entre 10 e 14 anos de idade, de cor negra, em situação de pobreza, proveniente de famílias numerosas e ambiente doméstico instável . Além disso, o agressor geralmente é alguém do sexo masculino, que possui vinculo familiar ou é próximo à família, aproveitando-se da privacidade do contexto doméstico (Saad, Hashish, Abdel-Karim \& Mohammed, 2016; David et al., 2018).

Uma vez imerso nesse contexto de violência, crianças e adolescentes que são vítimas de violência sexual sofrem consequências que podem ser físicas, psíquicas e sociais a curto e longo prazo (Aydin et al., 2015). Assim, as vítimas podem desenvolver baixa autoestima, se tornarem retraídas e violentas, reproduzindo os comportamentos aprendidos e carregá-los por toda a vida (Apostólico, Nobrega, Guedes, Fonseca \& Egry, 2012). Além disso, algumas dessas consequências são manifestadas através de condutas de risco, incluindo uso de drogas, prostituição, envolvimento em relacionamentos íntimos abusivos, delinquência e automutilação (Flynn-O’Brien et al., 2016).

No cenário nacional e internacional atual, em virtude da pandemia causada pelo vírus SARS-CoV-2, adotou-se como uma das estratégias de enfrentamento o distanciamento e isolamento social, mantendo apenas os serviços essenciais em funcionamento, com medidas mais severas em meados de abril ( Menezes \& Amorim, 2020; Pereira et al., 2020; Cluver et al., 2020). Em consequência, um terço da população global está confinada e o fechamento de escolas impactou mais de 1,5 bilhão de crianças, trazendo mudanças em seu cotidiano (Cluver et al., 2020).

Assim, mudanças no cotidiano são observadas tais quais: restrições de locomoção, redução/perda de renda, isolamento, superlotação dos domicílios e altos níveis de estresse e ansiedade. Esta recente configuração da convivência social resulta no aumento da probabilidade de as crianças experimentarem abuso físico, psicológico e sexual em casa, particularmente aquelas que já vivem em famílias violentas ou disfuncionais (Organização das Nações Unidas para a Educação, a Ciência e a Cultura [UNESCO], 2020).

O isolamento social estabelecido nas cidades brasileiras pode ser um fator preocupante no que diz respeito ao aumento no número de casos de violência sexual infantojuvenil. De acordo com dados do Disque 100, número para denúncia do Ministério da Mulher, da Família e dos Direitos Humanos (MMFDH), mais de 70\% dos casos de abuso sexual contra crianças e adolescentes acontecem no âmbito familiar, e são praticados por parentes (Ministério da Mulher, Família e dos Direitos Humanos [MMFDH], 2020). Além disso, as notificações desses fatos podem vir a não acontecer, tendo em vista que as escolas, principais porta vozes na denúncia das violações, estão fechadas e a maior parte das crianças não consegue romper o ciclo de violência sozinha (Melo et al., 2020).

Nesse contexto, este estudo tem como objetivo comparar casos de violência sexual contra crianças e adolescentes no período de 2018-2020. Acredita-se que tal estudo poderá contribuir para um melhor entendimento de como a pandemia afetou a 
ocorrência de violência sexual contra crianças e adolescentes, ao tempo que produzirá subsídios na elaboração de políticas sociais para prevenção e enfrentamento desse fenômeno no novo cenário que se apresenta.

\section{Metodologia}

Trata-se de estudo documental, analítico, realizado através de dados presentes em laudos periciais arquivados no banco de dados do Instituto Médico Legal de Teresina-PI. As pesquisas analíticas estão voltadas para a determinação de qual é o fator causal associado a um dado fenômeno. Em termos de resultado, validam ou rejeitam as hipóteses ou pressupostos formulados e dão bases para outros estudos analíticos ou experimentais (Andrade \& Pegolo, 2020).

Considerou-se como critério de inclusão: laudos realizados no período de abril a junho de 2018 a 2020; com identificação de violência sexual em vítimas com idade entre 0 e 18 anos. Foram excluídos do estudo laudos não finalizados pelo perito ou cancelados por erro administrativo no cadastro.

A coleta de dados ocorreu através de formulário específico que continha variáveis relacionadas a dados sociodemográficos da vítima (idade e sexo) e dados do episódio de violência (ano da ocorrência; sexo do agressor; vínculo da vítima com o agressor e local em que ocorreu a violência). O autor foi o único investigador envolvido nas revisões de registros e na coleta e interpretação dos dados, garantindo assim o registro padronizado de todas as informações apresentadas.

Os dados foram submetidos a processo de dupla digitação, utilizando-se planilhas do aplicativo Microsoft Excel e posteriormente exportados e analisados no software Statistical Package for the Social Science (SPSS), versão 22.0. A fim de caracterizar a amostra foram realizadas estatísticas descritivas de frequência absoluta e relativa. Para verificar associação entre as variáveis qualitativas foi utilizado o teste exato de Fisher ou o Teste do Qui Quadrado de Pearson, adotando-se o nível de significância de 5\%.

A pesquisa foi aprovada pelo Comitê de Ética em Pesquisa, sob o parecer $\mathrm{n}^{\circ}$ 4.170.476. Os preceitos éticos estabelecidos na resolução n 466/12 e 510/16 do Conselho Nacional de Saúde no que se refere a zelar pela legitimidade das informações, privacidade e sigilo das informações, quando necessárias, tornando os resultados desta pesquisa públicos, foram considerados em todo o processo de construção do trabalho.

\section{Resultados}

Observa-se na Figura 1, a distribuição dos laudos de violência sexual contra crianças segundo o ano de ocorrência. Observa-se para o ano de 2020 um declínio de 44,8\% no quantitativo de laudos realizados quando comparados com o ano de 2019. 
Figura1. Distribuição dos laudos de violência sexual contra crianças segundo o ano de ocorrência. Teresina, 2020. (n=309).

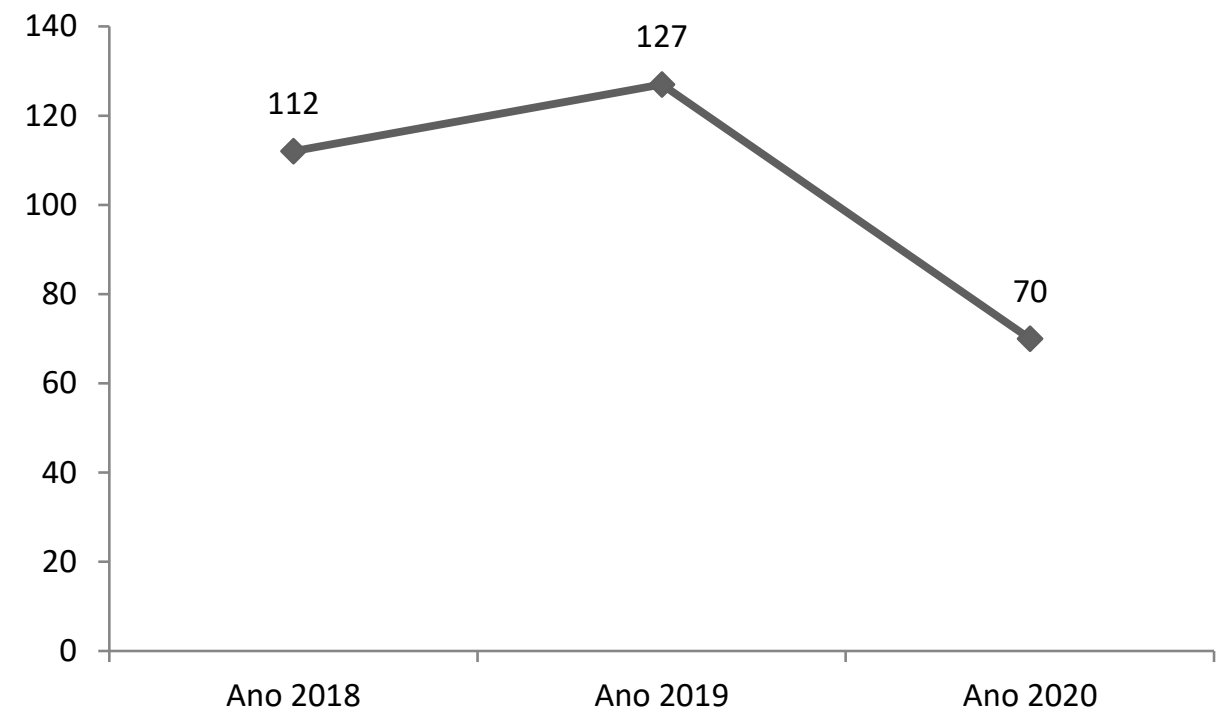

Fonte: IML Teresina.

De acordo com a Figura 2, quanto aos meses de ocorrência, observa-se no ano de 2018 uma manutenção no quantitativo de laudos; enquanto que no ano de 2019, há um aumento nos casos em maio e um decréscimo em julho; e em 2020, observa-se uma tendência de crescimento no decorrer dos meses.

Figura 2. Distribuição dos laudos de violência sexual contra crianças segundo o ano e mês de ocorrência. Teresina, 2020. $(n=309)$.

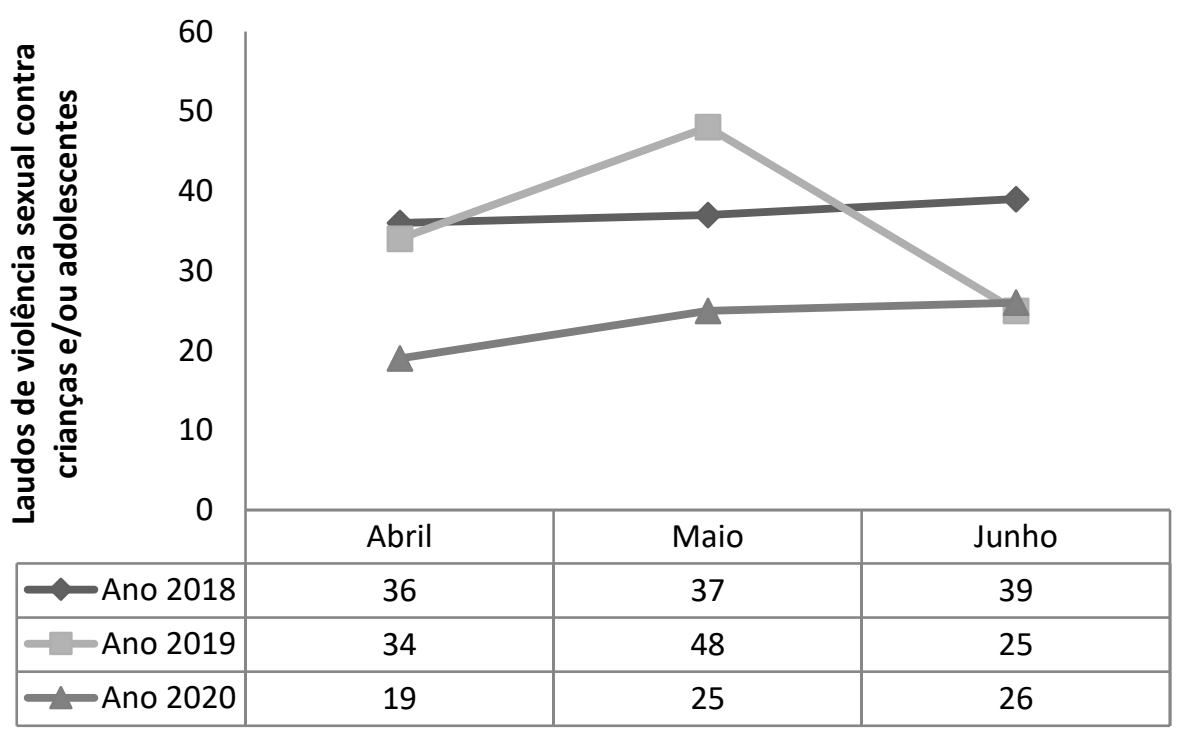

Fonte: IML Teresina.

A Tabela 1 apresenta a distribuição dos laudos de violência sexual em crianças e /ou adolescentes segundo as variáveis relacionadas à violência sexual. Observa-se um aumento no percentual de vítimas do sexo masculino e na faixa etária de 0 a 11 anos, quando se compara o ano de 2020 com os anos anteriores. Quanto ao sexo e número de agressores, aponta-se que agressores do sexo masculino e que agem sozinhos são predominantes. Quanto ao vínculo com o agressor e local da violência, observa-se 
no ano de 2020, um aumento no percentual de agressores intrafamiliar ( $\mathrm{p}=0,030)$ e o domicilio como local de ocorrência da violência. Em todos os anos, predomina como fonte de relato a própria criança e/ou adolescente vítima de violência.

Tabela 1. Distribuição dos laudos de violência sexual em crianças e /ou adolescentes segundo as variáveis relacionadas à violência sexual. Teresina, 2020. $(\mathrm{n}=309)$.

\begin{tabular}{|c|c|c|c|c|c|c|c|}
\hline \multirow{3}{*}{$\begin{array}{c}\text { Variáveis relacionadas à } \\
\text { violência sexual }\end{array}$} & \multicolumn{6}{|c|}{ Ano } & \multirow[t]{3}{*}{$p^{*}$} \\
\hline & \multicolumn{2}{|c|}{2018} & \multicolumn{2}{|c|}{2019} & \multicolumn{2}{|c|}{2020} & \\
\hline & $\mathrm{N}$ & $\%$ & $\mathrm{~N}$ & $\%$ & $\mathrm{~N}$ & $\%$ & \\
\hline Sexo da vítima & & & & & & & 0,467 \\
\hline Masculino & 13 & 11,6 & 11 & 8,7 & 10 & 14,3 & \\
\hline Feminino & 99 & 88,4 & 116 & 91,3 & 60 & 85.7 & \\
\hline Faixa etária & & & & & & & 0,195 \\
\hline 0 a 11 anos & 50 & 44,6 & 52 & 40,9 & 38 & 54,3 & \\
\hline 12 a 18 anos & 62 & 55,4 & 75 & 59,1 & 32 & 45,7 & \\
\hline Sexo do agressor & & & & & & & 0,354 \\
\hline Masculino & 104 & 92,9 & 119 & 93,7 & 62 & 88,6 & \\
\hline Feminino & - & - & - & - & 1 & 1,4 & \\
\hline Não informado & 8 & 7,1 & 8 & 6,3 & 7 & 10,0 & \\
\hline Número de agressores & & & & & & & 0,676 \\
\hline 1 agressor & 102 & 91,1 & 116 & 91,3 & 62 & 88,6 & \\
\hline 2 agressores & 2 & 1,8 & 3 & 2,4 & 1 & 1,4 & \\
\hline Sem informação & 8 & 7,1 & 8 & 6,3 & 7 & 10,0 & \\
\hline Vínculo com o agressor & & & & & & & $\mathbf{0 , 0 3 0}$ \\
\hline Intrafamiliar & 56 & 50,0 & 51 & 40,2 & 41 & 58,6 & \\
\hline Extrafamiliar & 48 & 48,0 & 68 & 53,5 & 21 & 30,0 & \\
\hline Não informado & 8 & 8,0 & 8 & 6,3 & 8 & 11,4 & \\
\hline Local da violência & & & & & & & 0,301 \\
\hline Domicilio & 43 & 38,4 & 45 & 35,4 & 33 & 47,1 & \\
\hline Escola & 5 & 4,5 & 2 & 1,6 & - & - & \\
\hline Casa de familiar & 16 & 14,3 & 11 & 8,7 & 10 & 14,3 & \\
\hline Via pública & 8 & 7,1 & 13 & 10,2 & 6 & 8,6 & \\
\hline Outros & 20 & 17,9 & 29 & 22,8 & 13 & 18,6 & \\
\hline
\end{tabular}




\begin{tabular}{lc|cccccc} 
Não informado & 20 & 17,9 & 27 & 21,3 & 8 & 11,4 & \\
Fonte do relato & & & & & & & 0,190 \\
Familiar & 24 & 21,4 & 27 & 21,3 & 20 & 28,6 & \\
Criança e/ou adolescente & 59 & 52,7 & 72 & 56,7 & 31 & 44,3 & \\
Testemunho de terceiro & 4 & 3,6 & 7 & 5,5 & 1 & 1,4 & \\
Suspeita por lesão genital & 7 & 6,3 & 5 & 3,9 & 6 & 8,6 & \\
Denúncia anônima & 18 & 16,1 & 25 & 11,8 & 9 & 12,9 & \\
Não informado & - & - & 1 & 0,8 & 3 & 4,3 &
\end{tabular}

* Teste do Qui Quadrado ou Teste Exato de Fisher, com nível de significância 0,05. Fonte: Autores.

\section{Discussão}

Este estudo possibilitou um comparativo na caracterização do perfil da violência sexual, com ênfase nos meses de isolamento social mais rígido devido à pandemia da Covid-19. Sabe-se que a violência sexual infantojuvenil é um problema de saúde pública não só no estado do Piauí, mas como em todo o mundo, principalmente em países subdesenvolvidos localizados na América do Sul, Ásia e África (Aydin et al., 2015; Silva et al., 2016; David et al., 2018; Francioli et al., 2018; Platt et al., 2018).

O conhecimento sobre a real magnitude da violência sexual infantojuvenil ainda não é satisfatório e a retratação dessa problemática ainda é escassa devido principalmente a subnotificação, má qualidade dos dados produzidos, pois muitos casos não chegam aos serviços de saúde e segurança pública, além disso, outros tantos chegam a ser velados, principalmente por que a vítima está muito próxima ao agressor (Almeida, Souza \& Sousa, 2007).

Nesse cenário, sabe-se que pandemia da COVID-19 transformou rapidamente o contexto em que crianças e adolescentes vivem. Medidas de quarentena, como o fechamento de escolas e restrições nos deslocamentos perturbaram a rotina e o suporte social das crianças (End Violence Against Children, 2020). Desta forma, o distanciamento social trouxe outros riscos relacionados ao próprio isolamento dentro do lar. Às vezes, a casa pode não ser uma opção tão segura para aqueles que já vivenciavam a violência doméstica. Isolar é uma tática de controle bastante utilizada pelos agressores, uma vez que, sem a rede social de apoio, é mais fácil manter a violência escondida dentro de casa (Fundo das Nações Unidas para a Infância [UNICEF], 2020c).

Considerando que, quando cometida contra crianças e adolescentes, a violência sexual geralmente tem caráter intrafamiliar e incestuoso, na qual os agressores são considerados cuidadores dos vitimados; acrescido ao fato de que cerca de 572 milhões de crianças e adolescentes em estão fora da escola devido ao fechamento das instituições de ensino, em 30 países, como iniciativa para a contenção de casos da COVID-19, evidencia-se uma posição de risco para esta população que, longe do espaço seguro que a escola oferece, passam maior parte do seu tempo agora em casa com seus agressores (Aydin et al., 2015; David et al., 2018).

Este estudo apontou redução no quantitativo de laudos periciais no ano 2020 quando comparado aos anos anteriores, em consenso com o apontado pelos relatórios do Disque 100, no qual o número de denúncias de violência sexual contra crianças e adolescentes no Brasil reduziu cerca de $12 \%$ entre março e junho de 2020 em comparação ao mesmo período do ano anterior (MMFDH, 2020). 
Informe epidemiológico sobre notificações de violência interpessoal ou autoprovocada no Distrito Federal, no período de abril a junho de 2020, mostrou que referente ao número de notificações por violência sexual houve um total de 682 notificações. Observou-se a predominância das ocorrências em adolescentes de 10 a 14 anos, com 151 notificações (22,1\% das notificações de violência sexual), no sexo feminino, foram registradas 139 notificações em indivíduos com 10 a 14 anos $(92,1 \%$ nas notificações de violência sexual). No segundo trimestre, foram 135 notificações para violência sexual, o que representa redução de 69,3\% em relação ao trimestre anterior (Secretaria de Vigilância à Saúde [SVS], 2020).

Uma pesquisa realizada no estado de Santa Catarina com base nas notificações do SINAN, observa-se queda progressiva de violência infantojuvenil. Ao comparar os meses de janeiro e abril, constata-se diminuição de $42 \%$ no número de notificações de violência (Platt et al., 2020). Em contrapartida no Reino Unido, houve um aumento de quase $20 \%$ no quantitativo de denúncias (Morgan, 2020).

Ao se fazer uma breve análise sobre o assunto na internet, percebe-se que o aumento da violência sexual contra crianças e adolescentes durante o período de isolamento social tem sido observado por diversas instituições nacionais e internacionais (End Violence Against Children, 2020; Silva \& Oliveira, 2020; UNICEF, 2020c).

A redução observada no cenário do estudo, pode estar relacionada ao fato de ser a violência sexual um fenômeno silencioso e o aumento das ocorrências no período pandêmico não ser relatado devido à escassez de mecanismos de denúncia, como fechamento das escolas e redução dos serviços de saúde e assistência social (Lund \& Mânica, 2020; Aprile et al., 2020; Honda et al., 2020). A utilização de serviços de prevenção em abuso infantil durante o a pandemia de COVID-19, em Nova York, diminuiu drasticamente em comparação com os sete anos anteriores anteriores (Whaling et al., 2020). A subnotificação dos casos acaba sendo um efeito colateral do isolamento social (Melo et al., 2020).

Uma pesquisa realizada pela UNICEF (2020a, p.2) sobre o impacto socioeconômico da resposta à COVID-19 observou que 104 países relataram uma interrupção nos serviços relacionados à violência contra crianças e adolescentes. O gerenciamento de casos e as visitas domiciliares para os grupos de risco estão entre os serviços mais comumente atingidos (UNICEF, 2020a).

Estudos de epidemias e crises anteriores mostram impactos devastadores na notificação de violência contra crianças e na prestação de serviços relacionados. Durante o surto de ebola na África Ocidental, por exemplo, as estruturas de bem-estar infantil e os mecanismos comunitários foram enfraquecidos, e as respostas de proteção à criança foram atrasadas ou afetadas (UNICEF, 2020c). Durante pandemias de saúde como a de Covid-19, o contato limitado com redes informais de apoio, como amigos, professores, cuidadores de crianças, parentes e membros da comunidade, deixa crianças e famílias mais vulneráveis (UNICEF, 2020c). Os mecanismos de notificação permitem que as crianças em perigo procurem ajuda e os mesmos devem ser adaptados aos desafios da COVID-19 (World Health Organization [WHO], 2020).

Sem o encontro com os colegas, sem a praça ou o centro de saúde, mas principalmente sem escola, o silenciamento é instalado. Atualmente, crianças e adolescentes encontram-se privados de contatos com redes de apoio extrafamiliares e escolas, isso dificulta o acesso a espaços de saúde e serviços específicos de proteção à criança (Lund \& Mânica, 2020).

Um relatório da UNICEF alerta sobre as consequências significativas e crescentes da pandemia de COVID-19 para crianças e adolescentes. Nele, estima-se que mais 15\% das crianças, cerca de 150 milhões, passaram a viver em pobreza multidimensional, ou seja, sem acesso à educação, saúde, habitação, nutrição, saneamento ou água (UNICEF, 2020b). A soma de todas as mudanças familiares, econômicas e sociais durante este período pandêmico pode se transformar em um potencial cenário favorável ao abuso sexual infantojuvenil, portanto, profissionais precisam ser vigilantes para esses fatores de risco (Teo $\&$ Griffths, 2020).

Com relação a caracterização sociodemográfica deste estudo, verificou-se que o sexo mais vitimado foi o feminino. Este dado coincide com outros estudos e reforça as evidências da fragilidade feminina nas relações de gênero, seja àquela decorrente da condição de superior força física do sexo masculino, da desigualdade expressa nas relações de gênero e da 
vulnerabilidade e risco da mulher para vitimização por violência, sobretudo para crianças e adolescentes (Sena \& Silva, 2018; Silva et al., 2016; Francioli et al., 2018).

A análise dos exames periciais permitiu evidenciar um maior percentual de vitimização na faixa etária adolescente. É possível que isto ocorra devido a um maior amadurecimento deste grupo para denunciar a violência sexual, já crianças menores tem uma capacidade reduzida de relatar a violência devido ao nível linguístico e cognitivo e pela falta de capacidade para entender determinadas interações como abusivas (Soares et al., 2016).

Quanto à caracterização da autoria, observou-se predomínio de envolvimento de um autor, do sexo masculino nos três anos analisados. Semelhantemente, estudos nacionais e internacionais revelam que a maior parte dos casos de violência sexual infantil é perpetrada por um autor do sexo masculino (Platt et al., 2018; Ministério da Saúde, 2018).

Quanto ao vínculo da vítima com o agressor e local da ocorrência da violência observou-se uma mudança nos percentuais ao se comparar o ano de 2020 com os anos anteriores: predomínio de caráter intrafamiliar e aumento percentual de casos ocorridos no domicilio. $\mathrm{O}$ isolamento social pode ter contribuído para essa mudança, pois o confinamento tem submetido crianças e adolescentes vulneráveis a um longo período de contato familiar que cria condições perfeitas para uma falha de salvaguarda externa nos casos em que as crianças já são ou virão a ser vítimas ou testemunhas de violência doméstica (Morgan, 2020). Além disso, o aumento do tempo dentro do lar leva ao estresse parental, que pode favorecer as tensões e conflitos, gerando muitas vezes, episódios de violência (Marques et al., 2020).

Segundo dados do Disk 100, no Brasil, ano de 2019 ocorreram 17 mil denúncias de violência sexual contra crianças e adolescentes, $73 \%$ dos casos ocorreram na residência da vítima, e em 46\% esta vítima era adolescente (12 -18 anos) e do sexo feminino, (MMFDH, 2020) o que coincide com os resultados desta pesquisa.

Nesse período de mudanças em face da pandemia, é de extrema importância que o poder público, os serviços de proteção à criança e a comunidade garantam a elas um cuidado seguro, apropriado dentro e fora do ambiente familiar (UNICEF, 2020c). De acordo com as orientações do Conselho Nacional dos Direitos da Criança e do Adolescente (CONANDA), conselhos tutelares, serviços de saúde e demais serviços da rede de proteção devem implementar ações para enfrentar o aumento dos casos de violência contra crianças e adolescentes durante a pandemia da Covid-19 (Conselho Nacional dos Direitos da Criança e do Adolescente [CONANDA], 2020).

Assim, o governo deve garantir que os planos de prevenção e resposta da COVID-19 integrem medidas apropriadas à idade e sensíveis ao gênero para proteger todas as crianças da violência, negligência e abuso. Os serviços de proteção à criança devem ser designados como essenciais e ter recursos adequados. As linhas de apoio nacionais, conselheiros escolares e outros mecanismos de denúncias favoráveis às crianças permitem que elas, em dificuldades, busquem ajuda e devem ser adaptadas aos desafios da COVID-19 (WHO, 2020).

Como limitações deste estudo, destaca-se a impossibilidade de resgate de variáveis cujos dados não foram informados, devido à utilização de dados secundários. É importante destacar ainda que a pesquisa demonstra dados presentes nos registros de laudos periciais de abuso sexual infantojuvenil, porém estes dados apresentados equivalem apenas aos casos denunciados, não revelando quantitativamente a realidade local quanto a este problema, devido à subnotificação.

Entretanto, estudos deste tipo conferem maior visibilidade a esse problema e indicam a necessidade de ampliação de políticas públicas voltadas à prevenção e combate ao agravo, além do cuidado a vítimas de violência sexual.

\section{Considerações Finais}

O cenário desta pesquisa demonstra alta taxa de violência sexual infantojuvenil. Houve um declínio no número de exames periciais realizados no ano de 2020. Observa-se um aumento no percentual de vítimas do sexo masculino, com idade entre 0 e 11 e que sofreram o abuso sexual em suas residências. Com relação ao agressor, houve predomínio do sexo masculino, 
que agem sozinhos. Quanto ao vínculo do agressor com a criança ou adolescente observa-se um aumento do vínculo intrafamiliar no ano de 2020 .

\section{Referências}

Almeida, L. A. A., Souza, L. S., Sousa, K. A. A. (2017). Epidemiologia da violência infantil um estado do nordeste do Brasil: série histórica de 2007 a 2016. Rev Pre Infec e Saúde, 3(2): 27-33.

Andrade, S. M. O., Pegolo, G. E. (2020). A pesquisa cientifica em saúde: concepçãp, execução e apresentação (2a ed). Campo Grande: UFMS.

Aprile, M., Carlis, M. F., Barcos, A., Barros, S., Cuadro, S., Di Vito, A. (2020). Cuando el tapabocas no protege: Pandemia y abuso Sexual en las infancias. Red Sociales, Revista del Departamento de Ciencias Sociales 7. 38-45.

Apostólico, M. R., Nobrega, C. R., Guedes, R. N., Fonseca, R. M. G. S., Egry, E. Y. (2012). Características da violência contra a criança em uma capital brasileira. Rev. Latino-am. Enfermagem, 20(2). 1-8.

Aydin, B., Akbas, S., Turla, A., Dundar, C., Yuce, M., Karabekiroglu, K. (2015). Child Sexual Abuse in Turkey: An Analysis of 1002 Cases. J Forensic Sci, 60 (1), 61-65.

Cluver, L., Lachman, J. M., Sherr, L., Wessels, I., Krug, E., Rakotomalala, S., Blight, S., Hillis, S., Bachman, G., Gren, O., Butchart, A., Tomlinson, M., Ward, C. L., Doubt, J., McDonald, K. (2020). Parenting in a time of COVID-19. Lancet, 395:e64.

Conselho Nacional dos Direitos da Criança e do Adolescente. (2020). Recomendações do CONANDA para a proteção integral a crianças e adolescentes durante a pandemia do COVID-19.

David, N., Ezechi, O., Wapmuk, A., Gbajabiamila, T., Ohihoin, A., Herbertson, E. (2018). Child sexual abuse and disclosure in South Western Nigeria: a community based study. Afr Health Sci, 18(2), 199-208.

Flynn-O’Brien, K. T., Rivara, F. P., Weiss, N. S., Lea, V. A., Marcelin, L. H., Vertefeuille J, Mercy, J. A. (2016). Prevalence of physical violence against children in Haiti: A national populationbased cross-sectional survey. Child Abuse \& Neglect, 51, 154-62

Francioli, A. V. S., Neves, L. V. F., Santos, J. V. C., Souza, L. G., Miguel, E. R. A. (2018). Violência sexual na criança e no adolescente: perfil epidemiológico de região metropolitana de Maringá-pr. Revista Científica Ágape, 1(1).

Fundo das Nações Unidas para a Infância. (2020a). COVID-19 causa interrupção nos serviços de proteção a criança em mais de 100 países. https://www.unicef.org/brazil/comunicados-de-imprensa/covid-19-causa-interrupcoes-nos-servicos-de-protecao-da-crianca-em-mais-de-100-paises

Fundo das Nações Unidas para a Infância. (2020b). UNICEF faz apelo urgente para evitar “geração perdida” para a COVID-19. https://brasil.un.org/index.php/ptbr/101524-unicef-faz-apelo-urgente-para-evitar-geracao-perdida-para-covid-19

Fundo das Nações Unidas para a Infância. (2020c). No dejemos que los niños sean las víctimas ocultas de la pandemia de COVID- 19. https://www.unicef.org/argentina/comunicados-prensa/no-dejemos-ninosvictimas- ocultas-pandemia-covid19.

Honda, C., Yoshioka-Maeda, K., Iwasaki-Motegi, R. (2020). Child Abuse and Neglect Prevention by Public Health Nurses during the COVID-19 Pandemic in Japan. Journal of Advanced Nursing, 76(11): 2792-3.

Lund, R. G., Mânica, S., Mânica, G. (2020). Collateral issues in times of covid-19: child abuse, domestic violence and femicide. Rev Bras de Odontol Leg, 7(2), 54-69.

Marques, E. S., Moraes, C. L., Hasselmann, M. H., Deslandes, S., Reichenheim, M. E. (2020). Violence against women, children and adolescentes during the COVID-19 pandemic: Overview, contributing factors, and mitigating measures. Cad. Saúde Publica 36(4), e00074420.

Melo, B. D. P., Serpeloni, D. R., Kabad, F., Kadri, J. F., Souza, M.S., Rabelo, M., Moura, I. V. (2020). Saúde mental e atenção psicossocial na pandemia COVID19: crianças na pandemia Covid-19. FIOCRUZ/CEPEDES.

Menezes, J. B., Amorim, A. M. A. (2020). Os impactos do COVID-19 no direito de família e a fratura do diálogo e da empatia. Civilistica.com, 9(1): 1-38.

Ministério da Mulher, Família e dos Direitos Humanos (2020). Relatório Violência contra crianças e adolescentes: $2018 / 2019$.

Morgan, T. (2020). Coronavirus: Child abuse calls to NSPCC up 20\% since lockdown. BBCnews. https://www.bbc.com/news/uk-wales-52473453

Organização das Nações Unidas para a Educação, a Ciência e a Cultura. (2020). Covid-19 educational disruption and response. United Nations Educational, Scientific and Cultural Organization. https://en.unesco.org/themes/education-emergencies/coronavirus-school-closures

Organización de Las Naciones Unidas. (2020). Los niños deben ser protegidos de La pedofilia y el abuso exacerbado por la pandemia del coronavirus. https://news.un.org/es/story/2020/04/1472542

Organização Mundial da Saúde. (2015). Relatório Mundial sobre a Prevenção da Violência. https://nev.prp.usp.br/wp-content/uploads/2015/11/1579-VIP-Mainreport-Pt-Br-26-10-2015.pdf

Pinheiro P. S. (2018). Violence against children: a global report. Ciência \& Saúde Coletiva, 23(5), 1591-99.

Platt, V. B., Back, I. C., Hauschild, D. B., Guedert, J. M. (2018) Violência sexual contra crianças: autores, vítimas e consequências. Ciência \& Saúde Coletiva, 23(4), 1019-31. 
Research, Society and Development, v. 10, n. 1, e11710111384, 2021

(CC BY 4.0) | ISSN 2525-3409 | DOI: http://dx.doi.org/10.33448/rsd-v10i1.11384

Platt, V. B., Guedert, J. M., Coelho, E. B. S. (2020). Violência contra crianças e adolescentes: notificações e alerta em tempos de pandemia. Rev. Paul. Pediatr, $39,1-7$.

Protecting children during the COVID-19 outbreak: resources to reduce violence and abuse End Violence Against Children; 2020 https:// www.endviolence.org/protecting-children-during-covid-19-outbreak

Saad, S., Hashish, R. K. H., Abdel-Karim, R. I., Mohammed, G.F. (2016). Emotional, physical and sexual abuse and its psychological impact in children. International J Contempor Pediatr, 3(3), 760-7.

Secretaria de Vigilância em Saúde, (2018). Análise epidemiológica da violência sexual contra crianças e adolescentes no Brasil, 2011 a 2017. Bol Epidemiol, 49(27), 1-17.

Sena, C. A., Silva, M. A., Falbo Neto, G. H. (2018). Incidência de violência sexual em crianças e adolescentes em Recife/Pernambuco no biênio 20122013. Ciênc. saúde coletiva, 23(5), 1591-1599.

Silva, C. B., Bastos, B. L., Santos, C. R., Moraes, M. S., Possuelo, L. G. (2016), Profile characteristics of sexual violence in children and teenagers in Rio Grande do Sul. Revista de Epidemiologia e Controle de Infecção, 6(2), 65-74.

Silva, E. R., Oliveira, V. R. (2020). Proteção de crianças e adolescentes no contexto da pandemia da Covid-19: consequências e medidas necessárias para o enfrentamento. Ipea.

Soares, E. M. R., Silva, N. L. L., Matos, M. A. S., Araújo, E. T. H., Silva, L. S. R., Lago, E. C. (2016). Perfil da violência sexual contra crianças e adolescentes. $R$. Interd, 9(1), 87-94.

Teo, S. S., Griffths, G. (2020). Child protection in the time of COVID-19. Journal of Paediatrics and Child Health, 146(1): 838-40.

Whaling, K., Larez, N. A., Sharkey, J. D., Allen, M. A., Nylund-gibson. K. (2020). Reduced child maltreatment prevention service case openings during COVID19. Research Square, 1-13.

World Health Organization (2020). Joint Leaders' statement - Violence against children: A hidden crisis of the COVID-19 pandemic. https://www.who.int/news/item/08-04-2020-joint-leader-s-statement---violence-against-children-a-hidden-crisis-of-the-covid-19-pandemic. 